Vietnam Academy of Science and Technology
Vietnam Journal of Earth Sciences
http://www.vjs.ac.vn/index.php/jse

\title{
Improved method for hydrochemical exploration of mineral resources
}

\author{
Nguyen Van Luyen*1, Oleg G. Savichev1 ${ }^{1}$ Viktor A. Domarenko², Quach Duc Tin ${ }^{3}$ \\ ${ }^{I}$ Department of Hydrogeology, Engineering Geology and Hydrogeoecology, Tomsk Polytechnic \\ University, Tomsk, Russian Federation \\ ${ }^{2}$ Department of Geoecology and Geochemistry, Tomsk Polytechnic University, Tomsk, Russian Federation \\ ${ }^{3}$ Department of the Science, Technology and International Cooperation, General Department of Geology \\ and Minerals of Vietnam (GDGMV)
}

Received 09 January 2017. Accepted 10 April 2017

\begin{abstract}
The article deals with a method for hydrochemical exploration and poorly studied areas based on the simulation and statistical modeling of the hydrochemical field. The peculiarity of the method is a prospecting area spotting under the following conditions: (1) the maximal ratio between river basin in the Riverhead without evident channel network and the total river basin; (2) the river network and tectonic deformations maximum; (3) presence of low-flow rate sections with relatively sharp breaks in grade of the water surface (outflow of rivers from mountainous areas onto the sub-mountain plain, extended sections of channel multi-branching). A sampling of 2-3 samples of surface water, 2-3 samples of river bed sediments, and 2-3 samples of ground water is taken at prospective sections and contiguous territories and the chemical composition determined. The geo-informational analysis and obtained data are used to determine the parameters of the model of the area under study, a predictive assessment of the hydrochemical indicators for prospective sections is carried out, and a detailed examination is planned and performed. The expected reduction in the cost of exploration compared to currently used methods is approximately $20 \%$.
\end{abstract}

Keywords: Hydrochemical exploration, hydrochemical background, anomaly.

(C)2017 Vietnam Academy of Science and Technology

\section{Introduction}

The discovery of hydrochemical anomalies is one of the most important stages of geochemical exploration for mineral resources and solving a range of geo-ecological tasks (environmental impact assessments for construction, setting permitted water pollution

"Corresponding author, Email: Luyennv@mail.ru levels, and others), and typically comprises the taking and subsequent analysis of a large number of samples of water, bed sediment, soil, rock, and vegetation at points on a regular grid. For example, in the Russian Federation, the current recommendation when conducting geochemical surveys on a scale of 1:200.000 is to take samples each $4 \mathrm{~km}^{2}$ with the potential for increasing density to 1 point 
Nguyen Van Luyen, et al./Vietnam Journal of Earth Sciences 39 (2017)

per 1-2 $\mathrm{km}^{2}$ (Requirements..., 2002). The volume of samples increases significantly when performing work at a high level of detail, which results in the time and expenditure needed to perform the work increasing to the point that profitability is lost. But that is only a part of the problem in increasing the general effectiveness of predictive and exploratory geological and geo-ecological works, the key factor for resolution of which is improving the (express or implied) geochemical models on which any hydrochemical study method is based.

A fairly complete survey of such models, methods, and methodologies for hydrochemical exploration of mineral resources is provided in the literature (Barsukov et al., 1981; Kolotov, 1992; Kraynov, Ryzhenko, Shvets, 2004; Kopylova, Guseva, 2014; Domarenko, 2012; Polikarpochkin, 1976; Shvartsev et al., 2005). Without repeating previous publications, we note that two main concepts are typically considered. The first comprises the presence of a sufficiently strong source from some geological epoch (usually of transmagmatic origin) forming the primary geochemical halo. This source is typically camouflaged by the formation of various origins forming the medium for the secondary geochemical halo but may be detected if there is a certain ratio of erosion and accumulative processes. Where there is a significant prevalence of the first (erosion) and an insufficiently "strong" source of chemical elements and compounds, geochemical anomalies are not formed or are concentrated in the crust without the formation of a mineral resource deposit, and where the second (accumulative processes) prevails, the geochemical halo is spatially limited and/or very difficult to detect. In this case, hydrochemical exploration is limited to studying the hydraulic erosion formations and tracing migratory water flows, which often involve the migration of chemical elements in suspension and the movement of stream sediments, or, somewhat more rarely in solution (Shvartsev S.L et al., 1997). The most obvious example of the use of this kind of approach is the exploration for placer gold deposits in river valleys (Domarenko, 2012; Lavyorov, Patyk-Kara, 1997).

The second concept assumes that even absent a single source it is possible that accumulative processes may prevail over migratory processes, which (if maintained over an extended period of geological time) may result in the formation of geochemical anomalies, including the formation of mineral resource deposits (Shvartsev S.L, 2008). This prevalence is most commonly due to relatively sudden global or regional changes in geochemical conditions on a geological time scale, significantly more rarely it is the result of modern processes (many geologists effectively view the latter case as a variation of remote location of the principal source and transformation of the geochemical halos (Lavyorov, Patyk-Kara, 1997; Mezhelovsky et al., 2001; Levashov et al., 2010).

In both cases, the hydrochemical study methodology is based on consideration of geochemical processes and includes planning and conducting sampling, laboratory work, and assessment of "background" and "anomalous" concentrations, as a rule in accordance with the accepted a priori law of the distribution of probabilities. Usually, this is normal (Gaussian) or log-normal distribution and the key rule for detecting anomalies is exceeding the interval limits $\left(\mathrm{E}(\varphi(C))-k_{\alpha} \cdot \sigma(\varphi(C))\right.$; $\left.E(\varphi(C))+k_{\alpha} \cdot \sigma(\varphi(C))\right)$, where $E(\varphi(C))$ is the expected value of function $\varphi(C)$ of concentration $C$ (including case $\varphi(C)=C$ ); $\sigma(\varphi(C))$ is the standard deviation of $\varphi(C) ; k_{\alpha}$ - inverse normal distribution at the level of significance $\alpha$ (The Instruction..., 1965; Perelman, 1979; Davis, 1986). Differences between the approaches described above are mainly found in the choice of environmental components studied, the form of migration of their chemical 
elements, and density of the sampling grid, which depend on the scale of exploration.

The authors' work under consideration attempts to: (1) build a simulated statistical model of the formation of hydrochemical anomalies that is not contrary to either concept and (2) to use the model as the basis for the development of a hydrochemical exploration method capable of reducing the volume of sampling without reducing effectiveness.

\section{Theory and Basic model}

A mathematical model is a convenient tool for studying reality, based on taking key parameters and the relationships between the parameters that define a system as a whole and disregarding other factors on the basis of error analysis of the related elements and relationships. This definition is also simultaneously a formulation of the limits on use of models: (1) if there are changes in the system as a complex of defined functions corresponding to the structure formed in the specified conditions - a set of elements (at the level of physical development or conceptualization) and the relationships between them, then the model used to study it must also be changed; (2) modelling is no different from guesswork if the error in determining modelling parameters is comparable to or greater than the error in predictive estimates made using the models. Correspondingly, these limits also formulate the main principles of modeling: (1) the probability the model is not adequately realistic is more than zero; (2) the adequacy of the model is evaluated for the weakest link (Loucks D.P et al., 2005).

The hydrochemical study process often uses some simplified mass transfer equations, which in one-dimensional form may be written as follows:

$$
\frac{\partial C}{\partial t}+v \cdot \frac{\partial C}{\partial x}=\frac{\partial}{\partial x}\left(D \cdot \frac{\partial C}{\partial x}\right)+f(C),
$$

where $C$ - substance concentration in the water medium; $t$ and $x$ - time and space coor- dinates; $v$ - velocity of flow; $D$ - hydrodynamic dispersion coefficient; $f(C)$ - a function characterising hydrochemical processes in the system and the introduction of substances from outside the system (Kraynov, Ryzhenko, Shvets, 2004; Lerman, 1979; Lekhov, 2010; Benedini, Tsakiris, 2013). Equation (1) is usually used in conjunction with a flowchart of initial and boundary conditions and certain simplifications. These conditions very commonly follow two options, when considering: (1) a thermodynamic model, provided $f(C)=0$; (2) a hydrodynamic stationary model with maximum simplification $f(C)=0$ or $f(C)=-k_{C} C$ $\left(k_{C}-\right.$ a parameter essentially corresponding to specific speed of change $C$ ), which, in turn, is additionally simplified by excluding either diffusion or advective components.

Another extremely important aspect of simulation modeling is the choice of means of description $D$. Typically it is oriented on evaluating the parameters of equation (2):

$$
D=D_{m}+\lambda \cdot v
$$

where $D_{m}$ - molecular diffusion coefficient; $\lambda$ - dispersion parameter (dispersive-ness parameter). In many cases where hydrodynamic models are used, $D$ is taken as a constant, while $f(C)=0$, which effectively corresponds to the propagation of flow disturbance an unlimited distance and liquidation (or substantial weakening) of the impulse source. However, if it is considered in general as a non-linear function of $C$, then given the results of studies of heat disturbance propagation in a nonlinear environment (Martinson L.K et al., 1996), it is possible to note the ability to localize increased substance concentrations within a limited spatial area due to volume absorption, when the "warming wave" is replaced by a "cooling wave" changing the direction of the. For the studied element and other chemical elements also, the resulting concentration gradient is an important factor in the formation of the geochemical barrier, which cre- 
ates a stronger spatial localization effect for high concentrations in the geological medium.

A similar effect is displayed in the formation of the structure section of peat deposits when significant changes in humidity occur at the boundaries of active and inert layers that have a non-linear relationship with hydraulic conductivity, hydrodynamic dispersion of dissolved salts and the function $f(C)$ (Savichev O.G, 2015). It can be amplified by a significant reduction in oxygen access and, as a result, a change in the redox conditions, removal of low-solubility compounds by both chemical reaction and absorption processes involving the generation of a layer preventing substance diffusion and the infiltration of atmospheric precipitation (Shvartsev S.L, 2015; Lasaga A.C, 1995). This effect can also be preserved after very significant environmental changes (according to (Gamov M.I et al., 2012), when the upper and lower boundaries of coal seams are often characterized by the presence of layers with a higher content of a range of chemical elements).

Analysis of hydrochemical and hydrological monitoring observations of Eurasian rivers (Fadeev et al., 1989; Savichev, Nguyen, 2015) indicate that substance concentration $C$ in water flows is related to water discharge $Q$. The nature of this relationship may be shown upon analysis of the system of standard differential equations describing changes over time of $C$ and $Q$ :

$$
\begin{gathered}
\frac{d C}{d t}=k_{C} \cdot C, \\
\frac{d Q}{d t}=k_{Q} \cdot Q,
\end{gathered}
$$

Where $k_{C}$ and $k_{Q}$ - specific velocity of change in substance concentration and water discharge, respectively. The ratio of $k_{C}$ and $k_{Q}$ is generally a function of water mass and temperature travel, which means (5) can be written.

$$
\frac{k_{C}}{k_{Q}}=k_{0}+k_{1} \cdot\left(\frac{Q}{Q_{0}}\right)^{k_{2}},
$$

Where $k_{0}, k_{1}, k_{2}$ - empirical coefficients; $Q_{0}$ - water discharge corresponding to certain initial conditions. In the light of the above, and using chain rule differentiation of the complex function, we obtain equation (6):

$$
Z=X^{k_{0}} \cdot \exp \left(\frac{k_{1}}{k_{2}} \cdot\left(X^{k_{2}}-1\right)\right),
$$

Where $Z=C / C_{0}$ and $X=Q / Q_{0}$ - modular coefficients of concentration and water flow rate; $C_{0}$ - the substance concentration corresponding to certain initial conditions. If the ratio of $k_{C}$ and $k_{Q}$ changes little over time, expression (6) takes on a power-law relationship of $C$ to $Q$, which is widely used in hydrochemistry and close in significance to the indirect indicators of substance migration in water used in geochemistry (Savichev O.G, 2010-2015).

Equation (6) enables a description of the temporal changes in the chemical composition of natural waters relating to the corresponding fluctuations in water runoff at a specific outflow but is difficult to apply without additional conditions for describing the spatial changes. In the latter case, it appears better to use expression (7), which was derived in (Savichev O.G et al., 2014) as a result of resolving a simplified equation for substance travel primarily due to advective transfer, provided the drainage basin of a river with area $F$ can be presented as part of an annulus with an angle at centre $\gamma$ and radius $L$, and the water mass movement is from the edge sector to towards the centre of the reference circle.

$$
C_{0}=C_{0, U} \cdot \frac{Y_{0, U}}{Y_{0}} \cdot\left(\frac{F_{U}}{F}\right)^{k_{3}},
$$

Where $C_{0}$ and $Y_{0}$ - characteristic substance concentrations for the period of time under consideration and depth of runoff from a river basin with area $F ; C_{0, U}$ and $Y_{0, U}$ - substance 
concentration and depth of runoff from the section of the river basin with area $F_{U}$ at an upper course without a pronounced channel; $k_{3}$ - coefficient reflecting the conditions for transfer from the run off layer to the reference average depth of flow and the chosen time scale. Assuming that the geochemical anomaly is situated in the inaccessible territory at the river source, the use of equation (7) with known values for $\mathrm{C}_{0}$ allows a significant reduction of time and effort in the process of determining $\mathrm{C}_{0}, \mathrm{U}$ (Savichev O.G et al., 2005).

The presence of a large number of factors and the nature of the processes whereby geochemical anomalies are formed means that concentrations of substances in the geological medium can be treated as random amounts, the behavior of which can be described by one of the laws of the probability distribution. In geochemical practice, as noted above, normal and lognormal distributions are most commonly used for these purposes. However, a number of other approaches should not be overlooked, e.g., proposals to use gamma distribution when describing hydrochemical run off (Dolgonosov B.V et al., 2015). However, the most logical and, simultaneously, simple choice is lognormal distribution, on the basis of the following assumptions (Savichev O.G, 2010, 2015).

(1) Consideration is given to the waterrock system formed under the influence of natural and anthropogenic factors over the course of a statistically homogeneous period.

$$
\sigma(C) \approx\left(\left|k_{0}+k_{1}\right|\right) \cdot C_{0} \cdot C v(Q) \approx\left(\left|k_{0}+k_{1}\right|\right) \cdot C_{g} \cdot C v(Q)
$$

Absent data on timed water discharges (or average daily discharges in overwetting zone), the annual water runoff coefficient of variation, calculated empirically depending on the area of the drainage basin $F$ and the average specific discharge $M_{Q, a}$ can be used in formula (10) as a first approximation. In particular, with consideration for the formula of S.N. Kritsky and M.F. Menkel (following (Chebotaryov N.P, 1962)), expression (10) takes the form:
Individual components of this system are in quasi-equilibrium and characterized by $N_{s}$ chemical reactions, which, subject to (Garrels R.M et al., 1965; Grenthe I et al., 1997), may be combined into a single overall reaction corresponding to equations $(8,9)$ :

$$
\begin{gathered}
\Delta G_{T}=R \cdot T \cdot\left(\sum_{i=1}^{N_{s}} \ln \Pi_{i}-\ln K_{T}^{0}\right), \\
\ln C_{y} \approx b_{0}-\sum_{j}^{N_{s}} b_{j} \cdot \ln C_{j},
\end{gathered}
$$

Where $\Delta G_{T}$ and $K_{T}^{0}$ - the overall change in Heimholtz free energy and the overall equilibrium constant at a given temperature $T ; \Pi_{i}$ the overall production of active components involved in each reaction; $C_{y}$ - the concentration of the target substance; $b_{0}, b_{j}$ are constants.

(2) The total quantity of substance $N_{s}+1$ is highly significant, which with consideration for the law of large numbers allows the probability distribution for $\ln C$ (and the characteristic time of transformation of the substance subject to (3)) to be treated as normal, and for the concentration $C$, log-normal.

(3) The expected value of $E(C)$, based on $(6,9)$ provided probability $N_{s}^{-1}$ is approximately constant, approximates to the geometrical mean $C_{g}$, and the standard deviation (subject to Taylor series expansion) - to function of $C_{0}$ and coefficient of water discharge variation $C v(Q)$ :

$$
\sigma(C) \approx \frac{k_{4} \cdot C_{g}}{F^{0,06} \cdot M_{Q, a}^{0,27}} .
$$

Where $k_{4}$ - empirical coefficient.

Summarizing the data, we note three key aspects of the simulated statistical hydrochemical model under consideration. First, the parameters $C_{0}$ and $Q_{0}$ in $(5-7,9)$ may be interpreted as the expected value of substance concentrations and water flow rates. However, 
$C_{0 \approx} C_{g}$, and $Q_{0 \approx} Q_{a}$, where $C_{g}$ - the geometric mean; $Q_{a}$ - the arithmetic mean. Absent observational data, the geometric mean value of $C_{g}$, can be estimated in the assumption that for a statistically homogeneous period the expected value of the hydrochemical run off $G_{0}$ from a unit of area of the drainage basin with a pronounced channel network (at each moment of time $G=C \cdot Q$ ) should not vary significantly, that is:

$$
\frac{d}{d t}\left(\frac{G_{0}}{F}\right)=0
$$

or

$$
C_{g} \approx C_{g, s} \cdot \frac{M_{Q, s}}{M_{Q, a}} \approx C_{g, s} \cdot \frac{M_{Q, a}^{k_{4}}}{M_{Q, a}}=C_{g, s} \cdot M_{Q, a}^{k_{4}-1}
$$

Where $M_{Q, a}$ and $M_{Q, s^{-}}$the arithmetic mean water runoff module at the present time, and at the commencement of functioning of the studied geosystem; $C_{g}$ and $C_{g, s}$ - the geometric mean substance concentrations corresponding to $M_{Q, a}$ and $M_{Q, s}$.

Second, the deviation of substance concentration from $C_{0}$ is determined: (a) in time - by fluctuations in water runoff according to (6); (b) in space - by the degree of drainage of the territory with higher substance content $\left(C_{0, U}\right)$ in various components of the river network. Where $k_{3}$ is a positive value, the latter is directly proportional to the area of the drainage basin from a river source without a pronounced channel $F_{U}$ according to (7), as well as the contiguousness of the river network and tectonic structures, which can be estimated by variations $P(r \cdot f)-P(r) \cdot P(f)$, where: $P(r)$ - drainage network density, equivalent to the probability of channel migration of surface waters; $P(f)$ - density of distribution of tectonic faults within the river basin; $P(r \cdot f)$ - probability of the river network and tectonic faults coinciding.

Third, subject to (Alekseyenko V. A et al., 2005), the background concentration of substances in a body of water (in water or bed sediment) may be treated as an expected value and estimated by determining the confidence interval for the geometric mean after excluding anomalous concentrations Cex. The latter are estimated in accordance with condition (14), and an integrated procedure for determining hydrochemical background and anomalies:

$$
C_{e x}>C_{g} \cdot\left(1+\beta \cdot\left(\left|k_{0}+k_{1}\right|\right) \cdot C v(Q)\right),
$$

Where $\beta$ - the normal distribution quantile with probability $\alpha / 2 ; \alpha$ - the significance level. Subject to a minimum margin of error in determining water discharges and substance concentrations in the water medium, and the recommendations of (Rozhdesvensky, Chebotarev, 1974), it is appropriate to take $\alpha=5 \%$, respectively - $\beta \approx 2$.

Thus, the model of hydrochemical processes in the supergene zone in general described by the equations (6-9, 13-14), allows describing a condition and long-term changes of system "water - rock". This model corresponds to the key concept: the hydromineral complex is the genetically connected association of connections of the chemical elements formed in a direction to equilibrium in the system "water - rock" and controllable by water flow intensity (as the factor determining time and conditions of such interactions) (Kopylova Yu. G at el, 2014; Udodov P.A at al., 1962; Shvartsev S.L, 2005, 2008).

\section{Results}

\subsection{Method of hydrochemical exploration for mineral resources}

Adaptation of the simulated statistical hydrochemical model described above to hydro chemical study practices with consideration for previous research (Savichev et al, 2015) enabled the formulation of the following principal provisions and phases of a method of hydrochemical exploration for mineral resources in poorly or unstudied inaccessible territories:

Geo-informatic analysis of the studied territory is carried out to determine the following parameters: 
- Determination of sections with a relatively weakly pronounced channel network, determination of their area $\mathrm{FU}$ and the total area of the drainage basin $\mathrm{F}$;

- Determination of the denseness of the river network $P(r)$ - ratio of channel network length to area of the river basin, density of tectonic fault distribution $P(f)$ - ratio of total length of faults (according to geological map) to area of drainage basin under consideration, probability of coincidence of the river network and tectonic faults $P(r \cdot f)$ - ratio of the length of the river network coincidence with tectonic faults (subject to map scale and doubled margin of error in determining distance using the map) to the area of the drainage ba$\sin$ and calculation of differences $P(r \cdot f)$ $P(r) \cdot P(f)$;

- As a first approximation, the $N_{1}$ sections with maximum $F_{U} / F$ and $P(r \cdot f)-P(r) \cdot P(f)$ values are taken as the most promising for exploration; the perspective of sections is assessed from the viewpoint of the first concept (strong source);

- $N_{2}$ low-flow sections with relatively sharp changes in water surface grade (river outflow from mountains to plain, sections with extensive braiding) are determined; the perspective of these sections is assessed from the standpoint of both concepts (strong source and accumulative processes prevailing over substance migration);

- A list $N_{3}$ of prospective sections is formed following the rule:

$$
N_{3}=N_{1}+\eta \cdot N_{2},
$$

Where $\eta$ - expert evaluation of the desirability of performing exploratory works at the $\mathrm{N}_{2}$ sections based on the results of analysis of the location of resources formed under similar geographical conditions (analogy principle); for each $N_{3}$ water flow:

- The depth of runoff $Y$ or specific discharge $M_{Q}$ (where possible, the coefficient of the water discharge variation $C v(Q))$ is determined for the drainage basin as a whole, for the section of the drainage basin without a pronounced channel network, and for other territories using the methods accepted in hydrogeological practice (Mujumdar P.P et al., 2012); where it is not possible to reliably determine the change in water runoff layer for the territory, $Y / Y_{0}=1$ is applied;

- The period of time $\tau$ in which the water runoff is approximately equal to the long-term average is determined, with $C_{\tau} \approx C_{g}$;

- 2-3 water samples and 2-3 bed sediment samples, and if possible 2-3 water samples from the aquifer drained as much as possible by water flow are taken during the period $\tau$. At least one sample (No.1) from each of the indicated components (surface water, bed sediment, groundwater) must be situated on the section with a relatively weakly pronounced channel network $F_{U}$, one sample (No.2) from the outflow forming the boundary of area $F$. Efforts should be made to ensure that the samples are taken at sections of the drainage basin with differing water surface grades. Sample No.3 may be taken from a section with relatively sharp changes in grade. The water and bed sediment samples shall be taken and their chemical composition determined in accordance with regulatory documents, for example, in the Russian Federation in accordance with (Requirements to manufacture....);

- For known values of concentrations $C_{1}, C_{2}$ $\left(C_{3}\right)$ and the corresponding values of drainage basin area $F_{1}, F_{2}\left(F_{3}\right)$ back-calculation using formula (7) determines the coefficient $k_{3}$;

- The geometrical mean value of concentration $C_{g}$ is calculated in the outlet of the drainage basin with area $F$ using formula (13) and standard deviation using formulas $(10,11)$;

- Concentration $C_{0, U}$ is calculated at the drainage basin section at the river source without a pronounced channel and/or for sections with a sharp change in grade (for concentrations $C_{2}, C_{3}$ and $C_{g}$ ); if the derived value of $C_{0, U}$ conforms to condition (14), the said 
section is deemed to have maximum prospective in terms of mineral resource exploration; if condition (14) is not met, expert evaluations of the value of $C_{0, U}$ at which the section is considered to have prospective may be used (for example, by analogy);

- Detailed geological and geochemical studies of the designated sections with high $C_{0, U}$ values are planned and conducted with a greater sampling frequency of river bed sediments and other environmental components;

- The data obtained is used to calculate the geometric mean and standard deviation and test condition (14); a geological-economic assessment of the territory is performed in the event of anomalous concentrations.

Partial testing of the method was performed using data on the chemical composition of Northern Vietnamese water flows (Bac Kan province, Cho Don district, Red River and Thai Binh River drainage basins, namely the interfluvial area of major tributaries - the Gam River and Cau River). The geological structure of the studied area involves three structural levels lying on the pre-Paleozoic granite-metamorphic foundation of the lower structural stage and not penetrated within the area (Figure 1). The middle structural level is formed from large graben syncline with Ordovician-Silurian and Devonian sedimentation. The graben syncline structure is complicated by sub-isometric depressions filled with upper Triassic deposits in the south-western area of the territory. The sedimentation is penetrated by varied, complex structured intrusions of gabbro-granite series from the upper Paleozoic and Meso-Cenozoic stages of tectonic-magmatic activation. Tectonic structures of various ages and orientations create a mosaic/block structure in the district and are the main factor favoring development of the river network in the territory. The district metals profile is determined by a significant quantity of occurrences and small deposits of lead, zinc, iron, manganese, apparently stratiform (Dao Manh Tien, 1984).
The main study targets are: the Cau river: (section of upper stream) - a large tributary of the Hong River system; the Pho Day river (tributary of the Hong river) and its tributary the Pho Day river; the Ta Dieng river, which flows into the $\mathrm{Ba} \mathrm{Be}$ lake; the Ban Thi river (tributary of the Gam river) and its tributary the Che Ngu river (Figure 1). Nguyen Van Luyen took 10 river water samples from a layer $0.3-0.5 \mathrm{~m}$ below the surface on 14-16 February 2015 (with the concurrent measuring of water temperature, specific electrical conductivity, and $\mathrm{pH}$ ) using specially prepared containers. Laboratory work was performed at the accredited hydrochemical laboratory of Tomsk Polytechnic University (state accreditation number No. ROSS RU. 0001.511901 of 12.07.2011). The specific electrical conductivity, permanganate demand, $\mathrm{pH}$, and concentrations of $\mathrm{Ca}^{2+}, \mathrm{Mg}^{2+}, \mathrm{Na}^{+}, \mathrm{K}^{+}, \mathrm{HCO}_{3}^{-}$, $\mathrm{CO}_{3}{ }^{2-}, \mathrm{CO}_{2}, \mathrm{Cl}^{-}, \mathrm{SO}_{4}{ }^{2-}, \mathrm{Si}, \mathrm{NH}_{4}{ }^{+}, \mathrm{NO}_{2}^{-}, \mathrm{NO}_{3}^{-}$, $\mathrm{PO}_{4}{ }^{3-}, \mathrm{Fe}, \mathrm{Zn}, \mathrm{Cd}, \mathrm{Pb}, \mathrm{Cu}, \mathrm{Al}$ in the samples were determined.

According to a considered method on a digital map (in a format of MapInfo) of scale 1: 50.000 total areas $F$ of river basins and sections with a relatively weakly pronounced channel network $F_{U}$ have been determined. Calculation of extent of tectonic faults and sites of concurrences of river valleys and tectonic faults is executed on a digital geological map of scale 1:200.000 (concurrence was estimated on a curve bending around which was carried out on meanders of the river in view of an error of definition of a map distance at a rate of $0.5 \mathrm{~mm}$ in the specified scale).

As a result of the study, the results of which are described in more detail in the work of O. G. Savichev and Nguyen Van Luyen (Savichev O.G et al., 2015), it was proven that the highest concentrations of $\mathrm{Zn}$ and $\mathrm{Pb}$ were found in the waters of the River Ban Thi and upper part of the Day river (where the Cho Dien deposit was previously discovered at Ban Thi with $\mathrm{Pb}+\mathrm{Zn}$ reserves of approximate- 
Vietnam Journal of Earth Sciences 39(2), 167-180

ly 10 million tons with a content of $3-24 \%$, and the Bang Lung deposit with reserves of more than 5 million tons, $\mathrm{Pb}$ content up to $9.5 \%$ and $\mathrm{Zn}$ up to $4.25 \%$ ). These sections coincide with Devonian deposits, intensive tectonic faults, and are characterized by the highest values of $F_{U} / F$ and $P(r \cdot f)-P(r) \cdot P(f)$, with the strongest association with the ratio $F_{U} / F$ found for lead, and for the difference $P(r \cdot f)-P(r) \cdot P(f)$ - with Zn (Figure 2, 3).

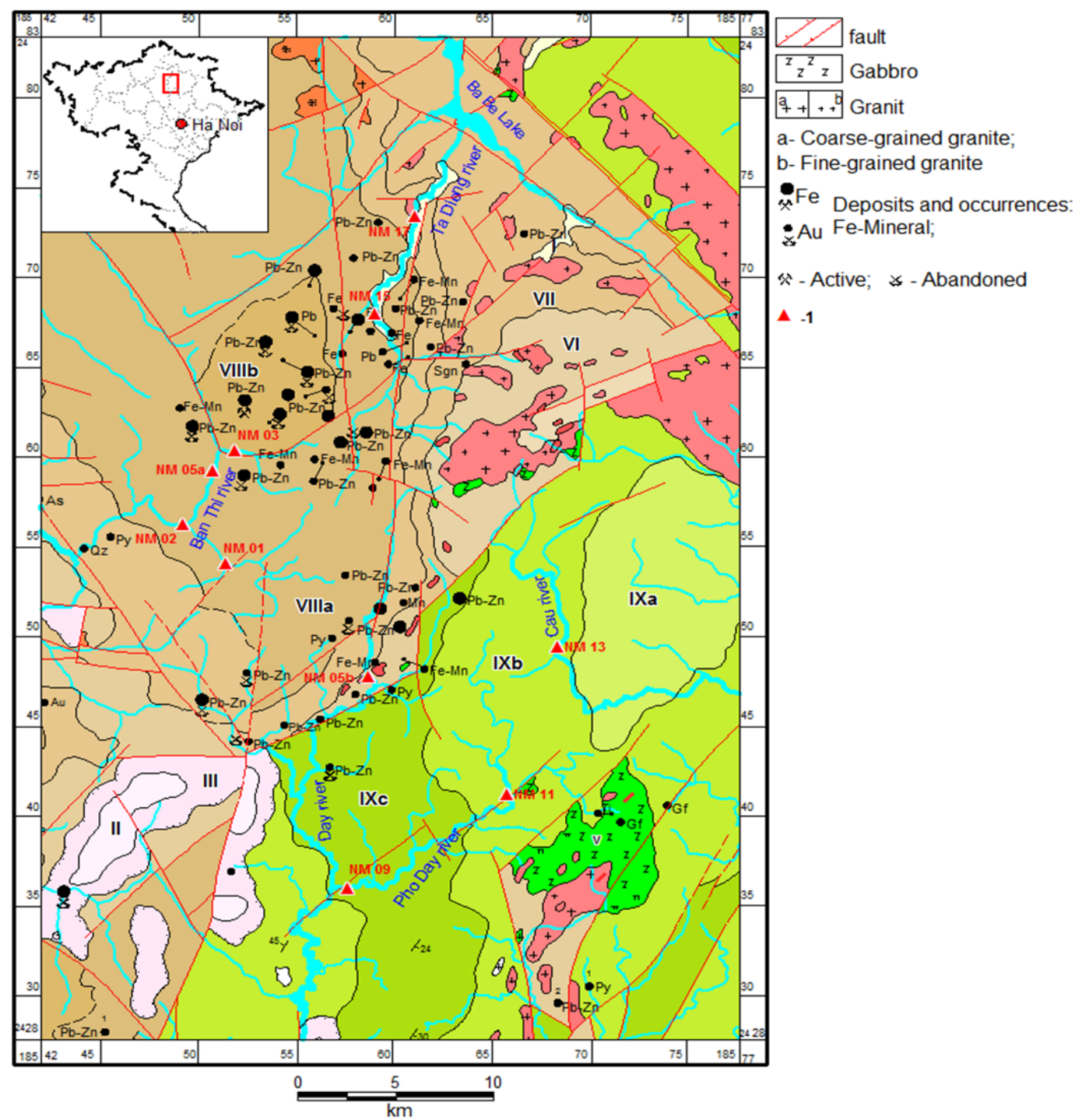

Figure 1. Geological structure of study area 1:200,000 (according to (Nguyen Kinh Quoc. 2001)), as amended), showing surface water hydrodynamic observation points (1): I - Undiscriminated Quaternary; II - Van Lang formation (upper subformation); III - Van Lang formation (lower subformation); IV - Van Lang formation (Phia Bioc complex); V - Van Lang formation (Nui Chua Complex); VI - Khao Loc formation; VII - Mia Le formation; VIII Pia Phuong formation (subformation: $\mathrm{a}$ - upper; $\mathrm{b}$ - lower); IX - Phu Ngu formation (subformation: $\mathrm{a}$ - upper; $\mathrm{b}$ middle; $\mathrm{c}$ - lower) 
Nguyen Van Luyen, et al./Vietnam Journal of Earth Sciences 39 (2017)

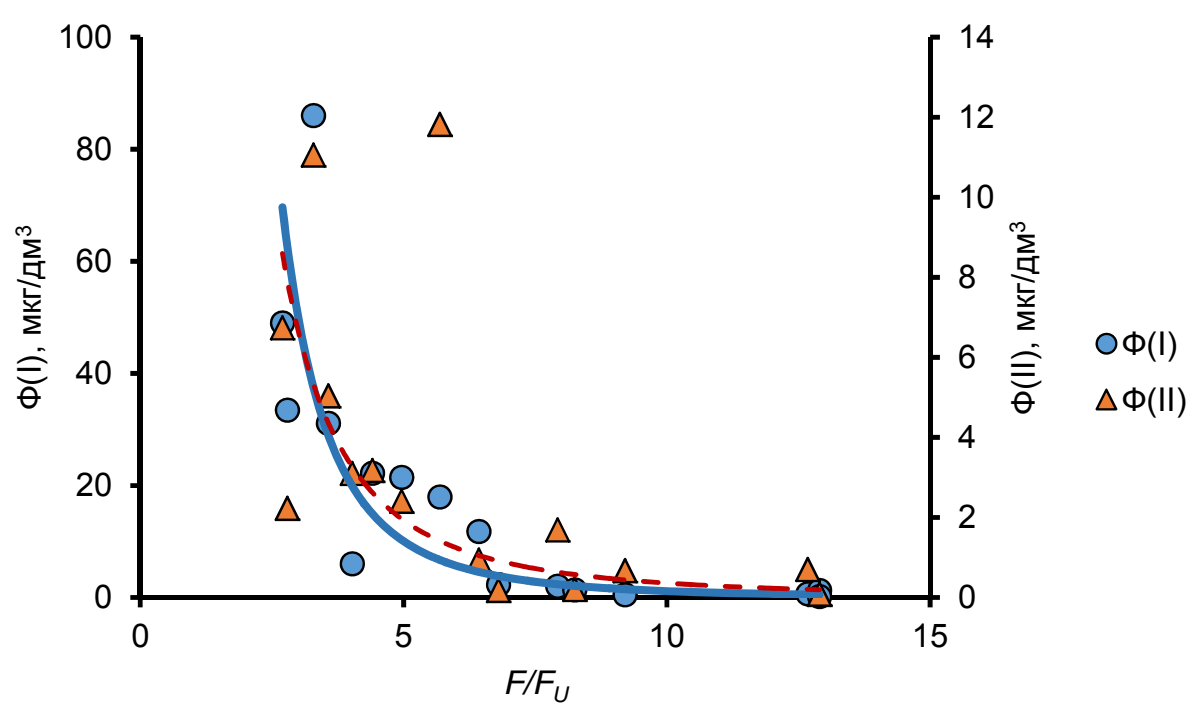

Figure 2. The relationship between $(\mathrm{C}) \mathrm{Zn}$ and $(\mathrm{C}) \mathrm{Pb}$ concentration and the ratio of the overall drainage basin area $\mathrm{F}$ and area of the upper without river network $\mathrm{F}_{\mathrm{U}}\left(\mathrm{Zn}=228.6 \cdot\left(\mathrm{F} / \mathrm{F}_{\mathrm{U}}\right)-1,35\right.$; the trend line: solid line of blue square $\Phi(\mathrm{I})=C(\mathrm{Zn}) \cdot Y / Y_{U}=1575,540 \cdot\left(F / F_{U}\right)^{-3,140}, R^{2}=0,83$; broken brown lines $\Phi(\mathrm{I})=C(\mathrm{~Pb}) \cdot Y / Y_{U}=95,211 \cdot\left(F / F_{U}\right)^{-2,422}$, $R^{2}=0,63$ correlation ratio $\mathrm{R}^{2}=0,39 ; \mathrm{Pb}=63.6 \cdot\left(\mathrm{F} / \mathrm{F}_{\mathrm{U}}\right)-1,81 ; \mathrm{R}^{2}=0,73$; critical value taken as $\mathrm{R}_{\text {lim }}{ }^{2}=0,36$ ) (according to (Statistical data of the People's Committee of Cho Don District), disregarding sample NM03, taken next to a factory); dotted line indicates trend; line colour corresponds to colour of $\mathrm{Zn}$ and $\mathrm{Pb}$ symbols

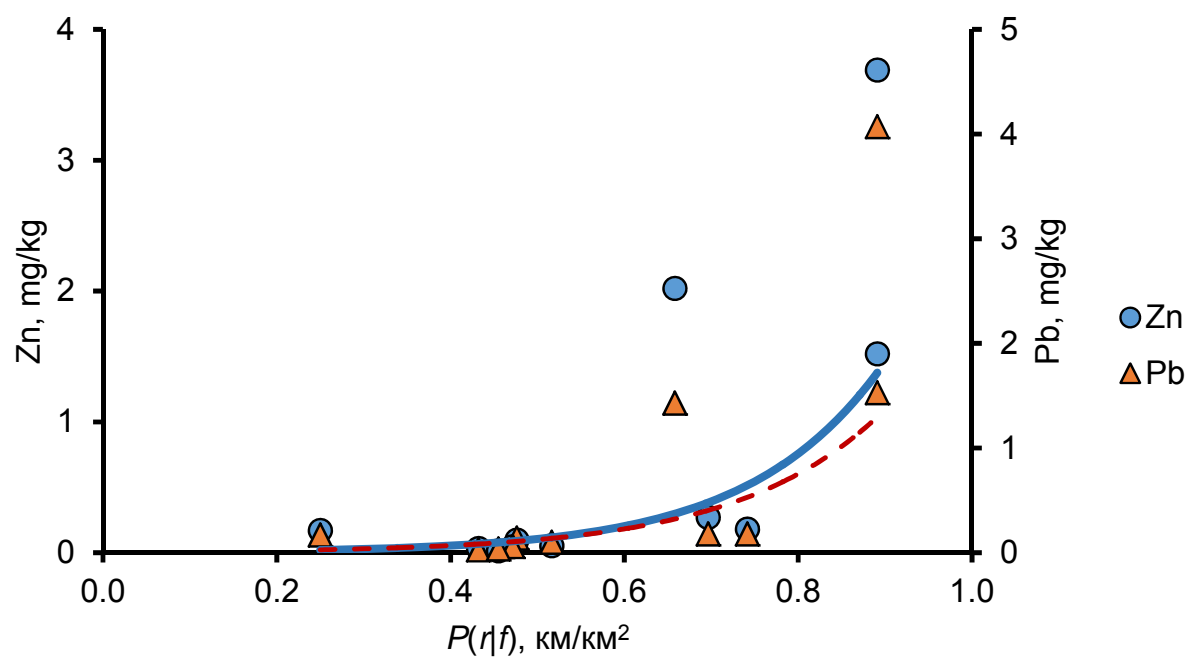

Figure 3. The relationship between $\mathrm{Zn}$ and $\mathrm{Pb}$ concentrations and the difference in probability of intersecting a tectonic fault $\mathrm{P}(\mathrm{r} \cdot \mathrm{f})$ and derived value $\mathrm{P}(\mathrm{r})$ and $\mathrm{P}(\mathrm{f})\left(\mathrm{Zn}=4.9 \cdot(\mathrm{P}(\mathrm{r} \cdot \mathrm{f})-\mathrm{P}(\mathrm{r}) \cdot \mathrm{P}(\mathrm{f}))+13.8 ; \mathrm{R}^{2}=0.81 ; \mathrm{Pb}=0.6 \cdot(\mathrm{P}(\mathrm{r} \cdot \mathrm{f})\right.$ $P(r) \cdot P(f))+1.6 ; R^{2}=0.68 ; R_{\lim }{ }^{2}=0.36$ ) (according to (Statistical data of the People's Committee of Cho Don District), disregarding sample NM03, taken next to a factory); dotted line indicates trend; colour of line corresponds to colour of $\mathrm{Zn}$ and $\mathrm{Pb}$ symbols 
Thus, changes of concentration $\mathrm{Zn}$ and $\mathrm{Pb}$ in river waters of researched territory as a whole are well described by the equation (7). Thus the maximal concentration $\mathrm{Zn}$ and $\mathrm{Pb}$ are observed on sites of known ore display of these metals for which ratio $F / F_{U}$ is minimal, and difference $P(r \cdot f)-P(r) \cdot P(f)$ is maximal. The physical sense of the received result consists the probability of formation of geochemical anomalies essentially increasing at the presence of a source of substance (access to which is realized on tectonic faults) and concerning low water migration. In result of such site accumulation of substance prevails in comparison with its carrying out. If similar situation is observed under rather constant conditions or the gradual reduction in intensity of water exchange during enough long time then it can lead to accumulation of the substance in the increased or high concentration. Certainly, it is only one of variants of the succession of events, but as has shown the analysis of the received data, its probability in some cases allows to use ratio $F / F_{U}$ and $P(r \cdot f)$ $P(r) \cdot P(f)$ as criteria of geochemical exploration for mineral resources.

\section{Discussions}

Thus, one of the key factors in the formation of the chemical composition of natural and natural-anthropogenic water is the intensity of water exchange, regulating the time and conditions of interactions in "water-rock" system (Lerman, 1979; Kraynov, Ryzhenko, Shvets, 2004; Shvartsev, 2008). The most important characteristics of the water exchange rate (in terms of its effect on the chemical composition of water) are the module of a water flow (water flow per unit time per unit area) and modular water flow rates (the ratio of water flow at a particular time or on average over a period of expectation).

The relationship between the modular coefficients of concentration and water flow rate looks as the function of the gamma distribu- tion (6). But the most part of observations usually corresponds to the recession curve, which looks as the inverse power dependence. A similar relationship is characteristic for the geometric mean hydrochemical indices, but with the norm (term average value) of module water flow. The most significant changes in the chemical composition of natural waters occur at the stage of the slope, subsurface and groundwater flow when the depending on the speed of movement of water generated the set of basic chemical reactions and physicalchemical processes, determining hydrochemical "background". At the stage of streamflow, this complex may vary, but not so much. Moreover, the standard deviation of hydrochemical indicators in direct proportion to the respective geometric mean and coefficient of variation of water flow (10). The latter value is inversely proportional to the area of the catchment (11). Respectively, it can be concluded that the variability of the concentration of the solutes decreases somewhat for large water bodies (both surface and underground) compared with smaller.

Another aspect of the impact of water flow on the chemical composition of water is to increase the content of substances at levels: (1) strengthening of the conjugation of the river network and tectonic disturbances; (2) decreasing in the ratio of general catchment area (in the numerator) for its part in the origins of the river without the expressed channel network (the denominator). Both features characterize the conditions of interaction of water with the rock (with primary aluminosilicate minerals and products of chemical reactions). Analysis of the data revealed a statistically significant relationship between the conditional probability confinement river network to tectonic disturbances $P(r \mid f)$ and the concentrations of substances in river waters and sediments, and also found an association between the condi- 
Nguyen Van Luyen, et al./Vietnam Journal of Earth Sciences 39 (2017)

tional probability $P(r \mid f)$ and empirical probability concentrations (Figure 4). Satisfactory convergence of the measured and calculated concentrations of $\mathrm{Zn}$ and $\mathrm{Pb}$ in river waters and sediments is achieved by using dependence (7).

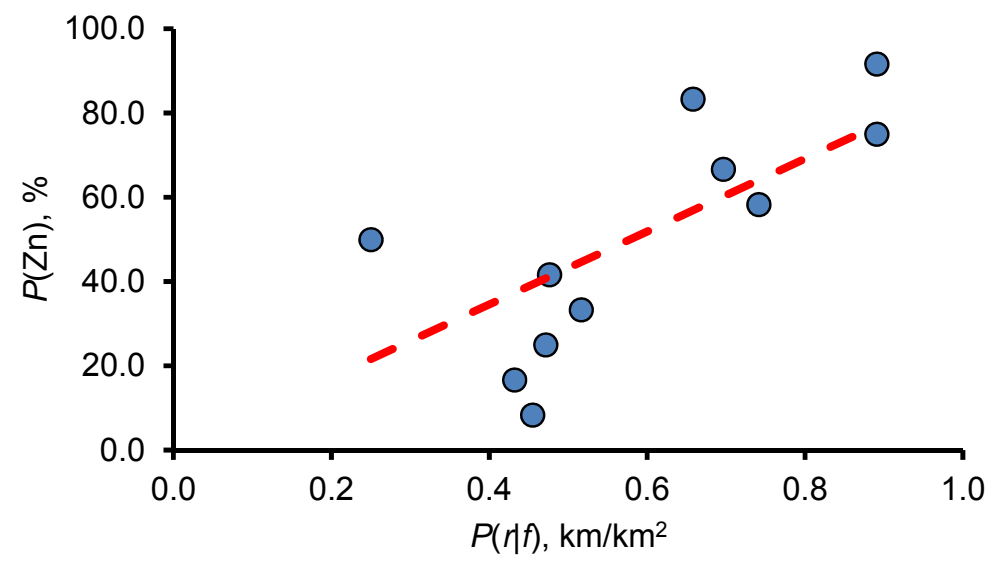

Figure 4. The relationship between the empirical probability of $\mathrm{Zn}$ concentrations in bed sediments and the conditional probability $P(r \mid f) ; P(\mathrm{Zn})=86.433 \cdot P(r \mid f), R^{2}=0.53$

In general, the catchment areas of the rivers studied, where mining is carried out leadzinc ores, items with elevated concentrations of $\mathrm{Zn}, \mathrm{Pb}$, and some other elements are associated, on the one hand, to the overlapping portions of the river network, part of the watercourses which is confined to the tectonic faults controlling the placement of lead-zinc occurrences and deposits, due to increased removal of chemical elements from the ore bodies. On the other hand, the increase in the concentrations of these elements with respect to the local geochemical background in general, the higher the larger the poorly drained catchment area in the vicinity of the manifestations and the closer is an anomaly of the enterprises for extraction and processing of ores.

Depending on the distribution of chemical elements in the water objects at different distances from the extraction sources and the enrichment of lead and zinc ores, the correlation coefficients between the conditional probability $P(r \mid f)$ and the content of heavy metal elements can be varied as: (1) $\mathrm{Zn}$ in river waters $0,73 \pm 0.16$; (2) $\mathrm{Zn}$ in extracted water from sediments $0,70 \pm 0.16$; (3) $\mathrm{Pb}$ in river waters $0,44 \pm 0.18$; (4) $\mathrm{Pb}$ in extracted water from sediments $0,69 \pm 0.16$. The high values are mainly found in the area near the mining areas.

Generally, the probability of detection of anomalous concentrations of $\mathrm{Pb}$ and $\mathrm{Zn}$ in sediments and river waters between the rivers Lo and Cau increased providing the rate of conjugation of the river network and tectonic disturbances $P(r \mid f)$ more $0,6 \mathrm{~km} / \mathrm{km}^{2}$, a the ratio of the catchment area and the upper part without the expressed channel network $F / F_{\mathrm{U}}$.

\section{Conclusion}

A simple simulated statistical model of the hydrochemical field is proposed, with the following key aspects: (1) concentrations of substances in surface and ground water, and river bed sediment are generally treated as random values subject to a lognormal probability distribution; (2) the hydrochemical background 
is taken as the expected value of the indicator for a section with approximately homogeneous geological landscape and hydrological conditions and calculated as the geometric mean; (3) fluctuations in concentrations relative to the background values have a nonlinear correlation with the modular coefficient of water flow, and the standard deviation is directly proportional to the hydrochemical background and coefficient of variation of the water runoff.

A method for hydrochemical exploration and poorly studied areas is proposed on the basis of this model. The distinguishing feature of this method is the determination of prospective sections using the following criteria: (1) maximum ratio of the area of the drainage basin at a river source without a pronounced channel network to the total area of the drainage basin; (2) maximum association of the river network with tectonic faults; (3) presence of low-flow rate sections with relatively sharp water surface grade changes (outflow of rivers from mountains to plain, extensive sections with braiding). 2-3 surface water samples, 2-3 river sediment samples, and 2-3 ground water samples are taken at promising sections and contiguous territories, and the chemical composition determined. The obtained data and data from geoinformation analysis of the studied area are used to determine the model parameters, a predictive estimate of hydrochemical indicators is made for the prospective sections, detailed studies are planned and conducted.

Calculations for the rivers of Siberia and North Vietnam (Savichev O.G et al, 2014, 2015) showed that FU/F typically does not exceed 0.2. Therefore, when using the proposed method, the number of samples taken, in particular, and the general cost of exploratory works (compared to the method currently used in the Russian Federation) would be reduced by approximately $20 \%$. Thus, general efficiency of search and exploration of miner- al resources will essentially increase and load on an environment will decrease as a result of carrying out of similar works.

\section{References}

Alekseyenko V.A, 2005, Geochemical methods of ore deposits searches, Logos, Moscow. In Russian, $354 \mathrm{p}$.

Barsukov V.L, Grigoryan S.V, Ovchinnikov L. N, 1981. Geochemical methods of searches of ore deposits, Nauka, Moscow. In Russian, 318p.

Benedini M., Tsakiris G, 2013. Water Quality Modelling for Rivers and Streams, Springer, Dordrecht, 287p.

Chebotaryov N.P, 1962. Theory of stream runoff, Moscow State University, Moscow. In Russian, 464p.

Dao Manh Tien, 1984. Methodology and features of geochemical specialization granitoide formations of Northern Vietnam, Azerbaijan State University, Baku. In Russian, 198p.

Davis J. C, Statistics and data analysis in geology. $2^{\text {nd }}$ edition, 1986, J. Wiley\&Sons, Toronto, 266p.

Dolgonosov B.V, Korchagin K.A, 2005. Probabilitical laws of the hydrochemical phenomena, Water resources, 4, 452-458.

Domarenko V.A, 2012. Rational a technique of searches and a geology-economic estimation of ore deposits of rare and radioactive elements. Vol.1, Prediction. Exploration and Evaluation, Tomsk Polytechnic University Publishing, Tomsk. In Russian, 167p.

Fadeyev V.V, Tarasov M.P, Pavelko V.L, 1989. A dependence of a total dissolved substancies and ionic composition of water of the rivers from their water regime, Hydromet, Leningrad. In Russian, 173p.

Gamov M.I, Granovskaya N.V, Levchenko S.V, 2012. Metals in a coal. South Federal University, Rostov on Don, Russia, 45p.

Garrels R.M, Christ C.L, 1965. Solution, minerals and equilibria, Freeman, Cooper, San Francisco. 450p.

Grenthe I, Puigdomenech I, 1997. Symbols, standards and conventions, in: Modelling in aquatic chemistry. Nuclear energy agency, Paris, 35-68.

Kolotov B.A, 1992. Hydrogeochemistry of ore deposits, Nedra, Moscow. In Russian, 192p.

Kopylova Yu.G., Guseva N.V, 2014. Hydrogeochemical methods of searches of ore deposits, Tomsk Polytechnic University Publishing, Tomsk. In Russian, 179 . 
Nguyen Van Luyen, et al./Vietnam Journal of Earth Sciences 39 (2017)

Kraynov S.R, Ryzhenko B.N, Shvets,V.M, 2004. Geochemistry of ground waters. Theoretical, Applied and Environmental Aspects, M: Science, Moscow. In Russian, 677p.

Lasaga A.C, 1995. Fundamental approaches in describing mineral dissolution and precipitation rates, Reviews in Mineralogy. Chemical Weathering Rates of Silicate Minerals, Mineralogical Society of America, 31, 23-86.

Lavyorov N.P. and Patyk-Kara N.G, 1997. Loosing ore deposits of Russia and countries of SNG, ed., Nauchny Mir, Moscow. In Russian, 453p.

Lekhov A.V, 2010. Physical-geochemical hydrodynamic. KDU, Moscow. In Russian. 500p.

Lerman A, 1979. Geochemical Processes Water and Sediment Environments, Wiley-Intersience Public, New York, 481p.

Levashov S.P, Yakymchuk N.A., Korchagin I.N., Bozhezha D.N, 2010. Operative estimation of the ore-bearing prospects of the license areas and the areas of operating mines and ore deposits, Geoinformatika (Ukraina). In Ukr./Rus, 4, 23-30.

Loucks D.P, Van Beek E, 2005. Water resources systems planning and management. An Introduction to Methods, Models and Applications, UNESCO Publishing, Turin, 680p.

Martinson L. K, Malov Yu. I, 1996. Differential equations of mathematical physics. IGTU of N. E. Baumann Publishing, Moscow, XII (1996) 1-368. In Russian.

Mezhevelovsky N. V. and Smyslov A.A., 2001. Mineral wealth of Russia. Vol.1, Mineral Resources, ed.. Mining Institute ICGC, Saint Peterburg - Moscow. In Russian, 285p.

Mujumdar P.P, Kumar D.N, 2012. Floods in a Changing Climate. Hydrologic Modeling, Cambridge University Press, New York, USA, 177p.
Nguyen Kinh Quoc, 2001. The Map of geological conditions and mineral resources in scale 1:200,000 of Bac Kan province, sheet F48-XV, Main Department of Geology and Minerals of Vietnam, Hanoi. In Vietnamese.

Perelman A.I, 1979. Geochemistry, high school, Moscow. In Russian, 423p.

Polikarpochkin V.V, 1976. Secondary auras and streams of dispersion, Science, Novosibirsk. In Russian, $407 \mathrm{p}$.

Requirements to manufacture and results multi-purpose geochemical mapping of scale 1:200.000, IMGRE, Moscow, 2002. In Russian.

Rozhdestvensky A.V, Chebotaryov A.I, 1974. Statistical methods in a hydrology, Hydromet, Leningrad. In Russian, $424 \mathrm{p}$.

Savichev O. G, Domarenko V. A, 2014. Laws of change of the chemical composition of river sediments and their use in searches of minerals, Fundamental research, 6, 520-525. In Russian.

Savichev O.G, 2010. Discharge regulation in surface water bodies, Water: chemistry and ecology, Vol. 9, 35-39. In Russian.

Savichev O.G, 2015. Distribution of Inorganic Pollutants over the Depth of Upper Peat Deposit, Contemporary Problems of Ecology, 1, 118-124.

Savichev O.G, Nguyen Van Luyen, 2015. Hydroecological condition between the Gam and Kau rivers (Northern Vietnam), Bulletin of Tomsk Polytechnic University, 7, 96-103.

Savichev O.G, Nguyen Van Luyen, 2015. The technique of determining background and extreme values of hydrogeochemical parameters, Bulletin of Tomsk Polytechnic University, 9, 133-142. In Russian. 